\title{
Equivalence of effective superpotentials
}

\author{
Riccardo Argurio \\ Physique Théorique et Mathématique \\ and \\ International Solvay Institutes \\ Université Libre de Bruxelles, C.P. 231, 1050 Bruxelles, Belgium \\ email: Riccardo.Argurio@ulb.ac.be
}

\begin{abstract}
We show that the low-energy effective superpotential of an $\mathcal{N}=1$ $U(N)$ gauge theory with matter in the adjoint and arbitrary even treelevel superpotential has, in the classically unbroken case, the same functional form as the effective superpotential of a $U(N)$ gauge theory with matter in the fundamental and the same tree-level interactions, up to some rescalings of the couplings. We also argue that the same kind of reasoning can be applied to other cases as well.
\end{abstract}


Recent research on the exact vacuum structure of $\mathcal{N}=1$ supersymmetric gauge theories has highlighted the central role played by the effective glueball superpotential $W_{\text {eff }}(S)$, in particular due to the systematic techniques developed in [1, 2, 3] in order to compute it. For a review and a list of references, see 4].

The object of this note is to show that seemingly different theories can have an effective glueball superpotential $W_{\text {eff }}$ with exactly the same functional form. We will discuss in some detail the case of the $\mathcal{N}=1 U(N)$ gauge theory with a fundamental-antifundamental pair $Q, \tilde{Q}$, which shares an equivalent effective superpotential with the same gauge theory with matter $\Phi$ in the adjoint and an even tree level superpotential $W_{\text {tree }}$, in the unbroken, maximally confining $U(N)$ vacuum. The proof of the equivalence uses the generalized Konishi anomaly, more precisely its reduction to the "ordinary" Konishi anomaly in the case of interest.

A similar, but technically more involved, treatment could in principle be given for other cases with matter in other representations, for instance in the antisymmetric representation. In fact, the present investigation was spurred by the conjectured equivalence of the effective superpotentials in this latter case in [5], conjecture based on the orientifold parent-daughter relation between the theory with the adjoint and the one with the antisymmetric. For $U(3)$, it becomes a relation between theories with adjoint and with fundamental matter.

We now discuss the determination of $W_{\text {eff }}$ in the simpler case of the fundamental matter. We find a generalization of the superpotential obtained in [6], using the method outlined in [7], that is using the Konishi anomaly equation.

Having one flavour, and calling the meson operator $X \equiv Q \tilde{Q}$, the general $W_{\text {tree }}$ has a simple form:

$$
W_{\text {tree }}=m X+\frac{1}{2} \lambda X^{2}+\ldots=\sum_{k} \frac{1}{k} \lambda_{k} X^{k} .
$$

Such a superpotential can of course be the result of integrating out other fields, e.g. in the adjoint, with Yukawa-like couplings to the fundamental ones, as in the example discussed in [6].

The Konishi anomaly, basically the anomaly for the $U(1)_{Q}$ rotations, yields the following relations for the expectation values in a SUSY vacuum:

$$
\sum_{k} \lambda_{k} X^{k}=S
$$


with:

$$
S=-\frac{1}{32 \pi^{2}} \operatorname{tr} \mathcal{W}^{\alpha} \mathcal{W}_{\alpha}=\frac{1}{16 \pi^{2}} \operatorname{tr} \lambda^{\alpha} \lambda_{\alpha}+\ldots
$$

The relation (21) is then solved to obtain $X\left(S, \lambda_{k}\right)$. The reason why we can do this so easily is that, $X$ being a chiral operator, its powers have the factorization property, i.e. in a SUSY vacuum:

$$
\left\langle X^{k}\right\rangle=\langle X\rangle^{k}
$$

Since there is no ambiguity, we drop the explicit reference to the VEV.

Having $X\left(S, \lambda_{k}\right)$, we can compute the effective superpotential by integrating the equations:

$$
\frac{\partial W_{\mathrm{eff}}}{\partial \lambda_{k}}=\frac{1}{k} X^{k}
$$

Note that a coupling independent part of $W_{\text {eff }}$ is left undetermined (the VY piece), so we are determining this way what is called $W_{\text {pert }}$.

As an example, we can consider the case $\lambda_{1}=m, \lambda_{2}=\lambda$ and $\lambda_{k}=0$ for $k \geq 3$. Here solving for $X$ is simple enough, and one can also integrate to find a closed expression for $W_{\text {pert }}$. We have:

$$
X=\frac{m}{2 \lambda}\left[-1+\sqrt{1+4 \frac{\lambda S}{m^{2}}}\right]
$$

and:

$$
W_{\text {pert }}=-\frac{m^{2}}{4 \lambda}\left(1-\sqrt{1+4 \frac{\lambda S}{m^{2}}}\right)+S \log \frac{1}{2}\left(1+\sqrt{1+4 \frac{\lambda S}{m^{2}}}\right)+S \log m .
$$

In order to obtain the full $W_{\text {eff }}$, one needs to add a VY piece 8 reflecting the effective gauge dynamics at scales where the $S U(N)$ confines and the matter field is integrated out. Note that in order to do this one has to carefully extract from $W_{\text {pert }}$ any remaining $S$-dependence.

We now consider the case of the theory with adjoint matter, and an even tree level superpotential:

$$
W_{\text {tree }}=\sum_{k=2}^{n+1} \frac{1}{k} g_{k} \operatorname{tr} \Phi^{k}, \quad g_{2 l+1}=0 .
$$

The effective superpotential is also determined by the VEVs of gauge invariant operators:

$$
\frac{\partial W_{\mathrm{eff}}}{\partial g_{2 k}}=\frac{1}{2 k}\left\langle\operatorname{tr} \Phi^{2 k}\right\rangle
$$


In this case however it is more involved to determine the VEVs, since in general a relation like (4) is not available. Hence the Konishi anomaly related to simple $U(1)_{\Phi}$ rotations is not enough to determine all the VEVs needed to extract $W_{\text {eff }}$. We need to generalize the Konishi anomaly relations, as shown in 3 .

Here we will remark that in an unbroken $U(N)$ vacuum, there are actually factorization-like relations $\left\langle\operatorname{tr} \Phi^{2 k}\right\rangle \propto\left\langle\operatorname{tr} \Phi^{2}\right\rangle^{k}$. We proceed to show this purely in $\mathcal{N}=1$ language.

We thus introduce the objects which allow to write an infinite series of Konishi anomaly relations in closed form [3], the resolvents:

$$
R(z)=-\frac{1}{32 \pi^{2}}\left\langle\operatorname{tr} \frac{\mathcal{W}^{\alpha} \mathcal{W}_{\alpha}}{z-\Phi}\right\rangle, \quad T(z)=\left\langle\operatorname{tr} \frac{1}{z-\Phi}\right\rangle .
$$

In terms of the above, the anomaly relations read:

$$
R(z)^{2}=W_{\text {tree }}^{\prime}(z) R(z)+\frac{1}{4} f(z), \quad 2 R(z) T(z)=W_{\text {tree }}^{\prime}(z) T(z)+\frac{1}{4} c(z),
$$

where $W_{\text {tree }}^{\prime}(z)=\sum_{k=1}^{n} g_{k+1} z^{k}$, and $f(z)$ and $c(z)$ are polynomials of degree $n-1$.

The solution for $R(z)$ is simple enough:

$$
2 R(z)=W_{\text {tree }}^{\prime}(z)-\sqrt{W_{\text {tree }}^{\prime}(z)^{2}+f(z)} .
$$

The basic feature of the above solution is the number of cuts on the complex plane it has. This allows to translate, in general, the data within the polynomial $f(z)$ into the gluino bilinear VEVs for every unbroken low-energy gauge group:

$$
S_{i}=\frac{1}{2 \pi i} \oint_{C_{i}} d z R(z)
$$

where $C_{i}$ circles the $i$-th cut.

If we are now in the case that all the eigenvalues of $\Phi$ distribute around one classical value (say, $\Phi=0$ ), the gauge group $U(N)$ is unbroken, and we expect at the effective level only one glueball effective superfield $S$. This corresponds to having only one cut in the solution for $R(z)$. In other words, all the zeros of $W_{\text {tree }}^{\prime}(z)^{2}+f(z)$ must come in pairs except two. We can thus write:

$$
W_{\text {tree }}^{\prime}(z)^{2}+f(z)=g_{n+1}^{2}\left(z^{2}-a_{1}^{2}\right)^{2} \ldots\left(z^{2}-a_{\frac{n-1}{2}}^{2}\right)^{2}\left(z^{2}-b^{2}\right) .
$$


Since $W_{\text {tree }}(z)$ is even, the problem is invariant under reflection in $z$. We have thus taken an even $f(z)$ and imposed that for any zero there is a mirror zero under reflection.

We do not need to consider further the resolvent $R(z)$, since what we need in order to compute $W_{\text {eff }}$ are the first few terms in the expansion of $T(z)$. From the anomaly equations (11), we see that $T(z)$ is given by:

$$
T(z)=-\frac{1}{4} \frac{c(z)}{W_{\text {tree }}^{\prime}(z)-2 R(z)}=-\frac{1}{4} \frac{c(z)}{\sqrt{W_{\text {tree }}^{\prime}(z)^{2}+f(z)}} .
$$

We recall now the conditions on $T(z)$, basically fixing the respective size of the low energy gauge groups $U\left(N_{i}\right)$ :

$$
N_{i}=\frac{1}{2 \pi i} \oint_{C_{i}} d z T(z) .
$$

In the unbroken case, this means that the overall contribution (the residue at infinity) comes entirely from the unique cut, while all residues at the simple poles must vanish. This condition implies that $c(z)$ is such that it cancels all the simple poles, and enforces that $T(z) \sim \frac{N}{z}$ when $z \rightarrow \infty$. Thus, the conclusion is that in the unbroken case $T(z)$ takes a very simple form, depending on a single parameter $b^{2}$ :

$$
T(z)=\frac{N}{\sqrt{z^{2}-b^{2}}} .
$$

We have then a simple expression for the $\operatorname{VEVs}\left\langle\operatorname{tr} \Phi^{2 l}\right\rangle$, all in terms of the single parameter $b^{2}$ :

$$
T(z)=\sum_{k \geq 0} \frac{1}{z^{k+1}}\left\langle\operatorname{tr} \Phi^{k}\right\rangle=\frac{N}{z}\left(1-\frac{b^{2}}{z^{2}}\right)^{-\frac{1}{2}}=\frac{N}{z} \sum_{k \geq 0} \frac{(2 k) !}{4^{k}(k !)^{2}} \frac{b^{2 k}}{z^{2 k}} .
$$

This implies:

$$
\left\langle\frac{1}{N} \operatorname{tr} \Phi^{2 k}\right\rangle=\frac{(2 k) !}{4^{k}(k !)^{2}} b^{2 k}=\frac{(2 k) !}{2^{k}(k !)^{2}}\left\langle\frac{1}{N} \operatorname{tr} \Phi^{2}\right\rangle^{k} .
$$

These relations have already been obtained through factorization of the Seiberg-Witten curve [9, see for instance [10]. Note that these are not chiral ring relations, and that they are not satisfied by giving a classical VEV to $\Phi$. Indeed, (18) also implies $\left\langle\operatorname{tr} \Phi^{2 l+1}\right\rangle=0$. In the following, it is useful to define $u_{k}=\left\langle\frac{1}{N} \operatorname{tr} \Phi^{k}\right\rangle$. For instance, we have the relation:

$$
u_{4}=\frac{3}{2} u_{2}^{2}
$$


We can now use the relations (19) in order to solve for $u_{2}\left(S, g_{k}\right)$, using only the equation for the $U(1)_{\Phi}$ chiral anomaly:

$$
\sum_{k} g_{k}\left\langle\operatorname{tr} \Phi^{k}\right\rangle=2 N S,
$$

which can also be written as:

$$
\sum_{k} g_{k} u_{k}=2 S
$$

Inserting (19), we obtain:

$$
\sum_{k} g_{2 k} \frac{(2 k) !}{2^{k}(k !)^{2}} u_{2}^{k}=2 S .
$$

This means that the functional form of $u_{2}\left(S, g_{2 k}\right)$ is given by the functional form of $X\left(S, \lambda_{k}\right)$, by $^{1}$ :

$$
u_{2}\left(S, g_{2 k}\right)=X\left(2 S, \frac{(2 k) !}{2^{k}(k !)^{2}} g_{2 k}\right) .
$$

Using the above expressions to obtain $W_{\text {eff }}$ for the theory with the adjoint, it can be expressed in terms of $W_{\text {eff }}$ of the theory with the fundamental as:

$$
W_{\text {eff }}^{(\operatorname{Adj})}\left(S, g_{2 k}\right)=\frac{N}{2} W_{\text {eff }}^{(\text {Fund })}\left(2 S, \frac{(2 k) !}{2^{k}(k !)^{2}} g_{2 k}\right) .
$$

As an example, consider the theory with:

$$
W_{\text {tree }}=\frac{1}{2} g_{2} \operatorname{tr} \Phi^{2}+\frac{1}{4} g_{4} \operatorname{tr} \Phi^{4} .
$$

We have that:

$$
u_{2}=X\left(2 S, g_{2}, \frac{3}{2} g_{4}\right)=\frac{g_{2}}{3 g_{4}}\left[-1+\sqrt{1+12 \frac{g_{4} S}{g_{2}^{2}}}\right] .
$$

and:

$W_{\text {pert }}=-N \frac{g_{2}^{2}}{12 g_{4}}\left(1-\sqrt{1+12 \frac{g_{4} S}{g_{2}^{2}}}\right)+N S \log \frac{1}{2}\left(1+\sqrt{1+12 \frac{g_{4} S}{g_{2}^{2}}}\right)+N S \log g_{2}$.

\footnotetext{
${ }^{1}$ Since $S$ and $g_{k}$ will anyway appear in dimensionless combinations in $W_{\text {eff }}$, some numerical factors can actually be shifted from one variable to another, consistently with (22), without any change in the final result.
} 
This same superpotential can be obtained through a matrix model computation, using for instance the results of [11.

A similar reasoning should be used to compare also with the effective superpotential for other theories, namely the one with antisymmetric matter considered in [5]. In that case, one should further be able to analyze the multi-cut case (that is, when the gauge group is broken to two or more factors at low energies).

One can also use the techniques applied here to the adjoint theory in order to compute $W_{\text {eff }}$ in the more general case of a not necessarily even $W_{\text {tree. }}$ In the one-cut case, $T(z)$ will depend on two parameters, the two edges of the cut. We will thus be able to express all the gauge invariants $\left\langle\operatorname{tr} \Phi^{k}\right\rangle$ in terms of, say, the first two. A relation among the latter two can be found using the (traced) classical equations of motion. Eventually, the ordinary Konishi anomaly will give us an equation for the remaining invariant in terms of the couplings and $S$. One can check that it is possible to rederive in this way the effective superpotential of the theory with a cubic interaction (and hence the free energy of the related matrix model, as given in 11]).

A more formal consequence of the equivalence of the effective superpotentials (77) and (28), is also the functional equivalence of the free energies of the related (one-cut) matrix models. The adjoint case is simply related to the free energy of the matrix model with quartic potential computed in [11. The fundamental case on the other hand can be related to a matrix model with boundaries [6], which in turn can be translated to a matrix model with a logarithmic potential. It would be nice to understand this correspondence at the matrix model level.

\section{Acknowledgments}

I would like to thank G. Bonelli, G. Ferretti and especially A. Armoni for very useful discussions and feedback. This work is supported in part by the "Actions de Recherche Concertées" of the "Direction de la Recherche Scientifique - Communauté Française de Belgique", by a "Pôle d'Attraction Interuniversitaire" (Belgium), by IISN-Belgium (convention 4.4505.86) and by the European Commission RTN programme HPRN-CT-00131. The au-

thor is a Postdoctoral Researcher of the Fonds National de la Recherche Scientifique (Belgium). 


\section{References}

[1] R. Dijkgraaf and C. Vafa, "A perturbative window into nonperturbative physics," arXiv:hep-th/0208048.

[2] R. Dijkgraaf, M. T. Grisaru, C. S. Lam, C. Vafa and D. Zanon, "Perturbative computation of glueball superpotentials," Phys. Lett. B 573 (2003) 138 arXiv:hep-th/0211017.

[3] F. Cachazo, M. R. Douglas, N. Seiberg and E. Witten, "Chiral rings and anomalies in supersymmetric gauge theory," JHEP 0212 (2002) 071 arXiv:hep-th/0211170.

[4] R. Argurio, G. Ferretti and R. Heise, "An introduction to supersymmetric gauge theories and matrix models," arXiv:hep-th/0311066

[5] A. Armoni, A. Gorsky and M. Shifman, "An Exact Relation for N=1 Orientifold Field Theories with Arbitrary Superpotential," arXiv:hep-th/0404247

[6] R. Argurio, V. L. Campos, G. Ferretti and R. Heise, "Exact superpotentials for theories with flavors via a matrix integral," Phys. Rev. D 67 (2003) 065005 arXiv:hep-th/0210291.

[7] A. Brandhuber, H. Ita, H. Nieder, Y. Oz and C. Romelsberger, "Chiral rings, superpotentials and the vacuum structure of $\mathrm{N}=1$ supersymmetric gauge theories," Adv. Theor. Math. Phys. 7 (2003) 269 arXiv:hep-th/0303001.

[8] G. Veneziano and S. Yankielowicz, "An Effective Lagrangian For The Pure N=1 Supersymmetric Yang-Mills Theory," Phys. Lett. B 113 (1982) 231.

[9] M. R. Douglas and S. H. Shenker, "Dynamics of SU(N) supersymmetric gauge theory," Nucl. Phys. B 447 (1995) 271 arXiv:hep-th/9503163.

[10] F. Ferrari, "On exact superpotentials in confining vacua," Nucl. Phys. B 648 (2003) 161 arXiv:hep-th/0210135.

[11] E. Brezin, C. Itzykson, G. Parisi and J. B. Zuber, "Planar Diagrams," Commun. Math. Phys. 59 (1978) 35. 